\title{
Healthcare Encounters Reference End Time Point End
}

National Cancer Institute

\section{Source}

National Cancer Institute. Healthcare Encounters Reference End Time Point End. NCI

Thesaurus. Code C123966.

A point in time that indicates the conclusion of a healthcare encounter. 